\title{
QoS Aware Cross Layer for SIP Based VoIP Over Enhanced OLSR Routing Protocol in Hybrid Wireless Network
}

\author{
Steven Raj N, Dhananjay Maktedar
}

\begin{abstract}
To improve SIP signal execution in MANET, routing parameters must be powerfully balanced through SIP methods dependent on a set equal for execution improvement measurements to help the SIP signal framework. In this manner, the presentation of the Optimal Link State Routing Protocol (OLSR) is to be additionally improved. In MANET, vitality is a key anxiety for secure communication, making it conceivable to avoid enemies or childish hubs since the system. In term of this paper, the projected secure as well as QoS based energy aware multipath routing in MANET. In support of multipath path collection, we have provided the Optimal Link State Routing Protocol (OLSR) algorithm. Energy efficient multipath routes are designated on the system using this method. Afterward a quantity of transactions, a route may misplace its connection quality. Hence the optimal path is selected to the paths installed on the system utilizing the Spider Monkey Optimization (SMO) algorithm. At last the presentation measurements of our planned SMO-OLSR task are contrasted and the current OLSR. SMOOLSR used for hybrid wireless network for efficient communication. The reproduction results demonstrate that the presentation of our planned work, the packet delivery rate, the delay, and the packet fallout are improved over the existing work. This planned methodology is actualized on the foundation of NS2.
\end{abstract}

Keywords: Optimal Link State Routing Protocol (OLSR), Spider Monkey Optimization (SMO), MANET, Multipath Routing, Hybrid Wireless Network (HWN).

\section{INTRODUCTION:}

Recently, the advancement of wireless technology has enabled many networks with different access technologies to simultaneously merge and meet the needs and needs of users. In hybrid wireless networks (HWNs), different networks are integrated to provide better services to the user [1]. Available alternatives include multi-interface terminal mobile devices such as the IEEE 802.11 Wireless Local Area Network (WLAN) [2], the IEEE 802.16 Global Accessibility for Microwave Access (WiMAX) [3], satellite systems [4] and Bluetooth [5] is in a multicultural context. However, various issues such as authentication, quality of service (QoS), user mobility, and authentication must be considered during the design and development of HWNs [6] [7].

Revised Manuscript Received on February 05, 2020.

* Correspondence Author

Steven Raj N, Research Scholar GNDEC, Bidar, Visvesvaraya Technological University Belagavi, Karnataka India.

E-mail: stevenraj.cse@gmail.com

Dr. Dhananjay Maktedar, Professor GNDEC Bidar, Visvesvaraya Technological University, Belagavi, Karnataka India.

E-mail:dhan_mak@yahoo.com

(C) The Authors. Published by Blue Eyes Intelligence Engineering and Sciences Publication (BEIESP). This is an open access article under the CC BY-NC-ND license (http://creativecommons.org/licenses/by-nc-nd/4.0/)
The demand for multimedia services over wireless networks such as Video on Demand (VoT) or Voice over IP (VoIP) has increased tremendously over the past year. These multimedia services have some quality service (QoS) requirements that must be met. Support for Session initiation Protocol (SIP) is essential to provide multimedia services and voice over Internet protocol on different networks. It has diverse access advancements, covering and inclusion and conventions for arrange engineering, traffic, steering and portability of the executives. Diverse administrator commercial center offers distinctive assistance requests from clients like voice, video, sight and sound, content. Due to these variations, there is an essential to delegate the message network since one system to additional network as the user moves and considers its features and user needs. Uninterrupted mobility (user movement) of the HWN is one cause of the vertical handover problem [8]. Channel handover is done between the two different systems from the vertical signature. Many previously published researchers have dealt with the same problem with different heuristics measurements. Policy-based handover by incorporating mobility management into a policy-based framework to achieve signatures are stimulated not only by signal strength then the policy constraints like price, reserve obtain ability and load balance.

HWN is a combination of an infrastructure-based network where a mobile device can connect to a base station (BS) using wireless channels over other sensor nodes on the Internet. The main components of the WSN are the huge amount of sensor hubs accomplished of sensing, interacting and acting. These special nodes have limited processing and communication capability due to limited energy [9]. MANET is a set of wireless mobile devices, all of that can be mobile, that can switch to a wireless network without using any infrastructure. Mobile devices are powered by a battery, and battery power is restricted. Similarly, autonomous, sensor nodes detect instances of gathering information and sending the processed data in a powerblocked manner [10]. Trusted Framework for a Hybrid RPR-EPON-WiMAX system uses a service type-depend scheduling on together the EPON and WiMAX system with plots specific classes in the RPR to the defined provision categories on the WiMAX system [11].A multi-target matching difficult in accommodating sensor networks utilizing two-way time-arrival (TW-TOA) with TDOA measurements performed by primary and subordinate hubs [12]. An energy-balanced centralized with circulated mechanism for powerfully transmitting mobile sensors in a hybrid WSN [13]. 
The low-level routing technique is used for performance measurement basis for base stations with a single antenna, while providing better performance, easier operation than conventional programs [14]. The tree-based motion management system [15] is used to diagnose and solve a loop problem by monitoring and controlling the depth of the tree. This technique not only decreases the chance of loop creation, then it solves the loop rapidly.

\section{LITERATURE SURVEY:}

Yuan Chai et al. [16] have provided a current load with interrupt-balance hybrid routing protocol. LIP-HRP considers the load position on the interrupt hubs since the interference hubs through the highest load will have a greater influence on the current node. A high load level at the intersection point will bring about long-term interference. Besides, the energy level of the mesh customers is occupied into explanation in the planned method since the power of the mesh customers is low. The stimulation results demonstrate that LIB-HRP can accomplish to improved execution as far as normal bundle misfortune rate, postpone and organize execution and vitality utilization.

Chaudhariet al. [17] has proposed a central authority-created reserve forecasting instrument for mobility (CA-RPM) concept, which calculates resources utilizing representatives concluded a resource forecasting firm containing of a fixed mediator, a psychological specialist, and two portable operators. Operators can proficiently foresee traffic, portability, cradle space, vitality and data transfer capacity required for productive asset allotment to help ongoing and mixed media interchanges. Versatile operators gather and appropriate system traffic measurements by means of MANET, while a static specialist gathers neighborhood insights. CA makes a static/portable operator through the asset gauging process. At first, structured time arrangement data WNN anticipate traffic and versatility. Cushion space, vitality with transmission capacity anticipating use anticipated portability and transportation.

Santiago Gonzalez et al. [18] have evaluated measurable video streaming on a mobile ad hoc system by two programs. In this project, cinematic is spread by upholding a continuous exchange degree with transferring data across a total layer. The additional project involves an adaptive classic for the transport basis removes coatings since the SVC stream according to the accessible bandwidth. Two corresponding tools for analyzing SVC circulation for information systems. Routing protocol through interlayer messages to evaluate with communicate (based on available bandwidth) the available resources on the network.

Sagan Lal et al. [19] the aforementioned limitations of existing QoS-aware routing and the multiple barrier QoEcentered routing technique for effectively transmitting multimedia traffic on MANETs. In a large-scale simulation system, human-on the up equipment in-circle QoE evaluations and ongoing video transmissions utilizing interactive media programming with a responsive MANET routing protocols. The weighted metric-based link quality estimation system reflects the things of shadow with system flexibility through its QoE-focused route detection development. A two-dimensional mathematical classical has been introduced to calculate joint path-quality and pathdelay on candidate paths. A test bed (developed and captured by physical machines) used to test and evaluate the performance of real-time video streaming.

Ying Baoet al. [20] have provided the QoE assessment criteria for improving the quality of experience (QoE) based on the organize media transmission administration and for changing the transmission control system. In this manner, a sort of QoE composite estimation strategy dependent on fluffy bunching heuristic calculation is utilized, which centers around administration score computation on the server side. The server side gathers the system transmission QoS parameter, hub area information, and client desire an incentive from customer criticism data. It at that point deals with the chronicled information in the database through a "major information" process, and predicts client scores as per heuristic guidelines. On this premise, it finishes the vague grouping investigation and creates a help QoE score and the board message that will eventually be conveyed to clients.

Chettibiet al. [21] have proposed a dynamic fuzzy energybased AODV (DFES-AODV) routing protocol for MANET. In the DFES-AODV path discovery phase, Mamdani uses fuzzy logic structure (FLS) to determine the probability that each node shares its path requests (RREQs). FLS inputs are the remaining battery level as well as vitality channel pace of the versatile hub.

DeminGao et al. [22] familiarize a new routing protocol depend on a cross-technical communication method called Robi. Ruby uses integrations since the Wi-Fi hubs to help ZigBee hubs establish routing tracks that permits hubs to independently indicate their duty-cycles through the best duty sequence. A humble and efficiency technique is used so that the ZigBee nodes are synchronized to the beacons broadcast by the WiFi nodes. The problem statement and contribution of our proposed work is given as follows;

Movement of MANET hubs as well as associated routing problems growth the time compulsory for logging, call setup, in addition to call dissolution procedures, reflecting the overall presentation of SAN-depended VoIP / multimedia applications. Furthermore, the central environment of the SIP signal scheme is not appropriate for the MANET environment since of the movement of the hubs, the routing instrument, also the signal problems like noise, intrusion too fading. Hence, the path signal is gone, the SIP signal scheme is straight affected then the presentation of the VoIP submissions is reduced. Thus, to improve SIP signal presentation in MANET, routing measurements must be dynamically used to SIP procedures depended on a set side by side presentation improvement metrics to maintenance the SIP indicating scheme. Therefore, the performance of the OLSR is to be further improved.

Contribution of the proposed approach is described below:

- A cross-layer method for advanced OLSR routing algorithm is presented to improve the presentation used for the combination finished hybrid wireless network.

- Improving the performance of the OLSR routing protocol by improving multipoint relay nodes using the Spider Monkey Optimization (SMO) algorithm.

- The effectiveness of this planned method is appraised depended on the packet delivery rate, delay. 
This paper composed as pursues. Inquires about dependent on multipath steering in MANET are connected with our planned technique in section II. Multimedia applications over hybrid wireless network, a cross layer approach for enhanced OLSR routing algorithm and the performance of OLSR protocol is enhanced by optimally selecting the multipoint relay nodes using the spider monkey optimization algorithms are presented in section III. Consequences of our planned work are conferred in section IV. This broadside is determined through section.

\section{PROPOSED METHODOLOGY:}

Movement of MANET hubs also associated routing problems proliferation the time compulsory for logging, call setup, and call conclusion developments, reflecting the overall presentation of SIP-depend VoIP / multimedia submissions. Furthermore, the brought together nature of the SIP signal framework isn't appropriate for the MANET environment as a result of the development of the hubs, the routing system, and the sign issues, for example, clamor, impedance and blurring. Consequently, when the way signal is lost or postponed, the SIP signal framework is legitimately influenced and the presentation of the VoIP applications is decreased. Thus, to improve SIP signal presentation in MANET, routing measures must be dynamically used to through SIP procedures depend on a set near for presentation improvement metrics to maintenance the SIP signaling scheme. Therefore, the performance of the OLSR is to be further improved. In order to improve the performance for multimedia applications in a hybrid wireless system, in this investigation, a cross-layer approach for improved OLSR routing algorithm is proposed. The presentation of the OLSR algorithm is optimized by optimizing multipoint relay nodes using the spider monkey optimization (SMO) algorithm.

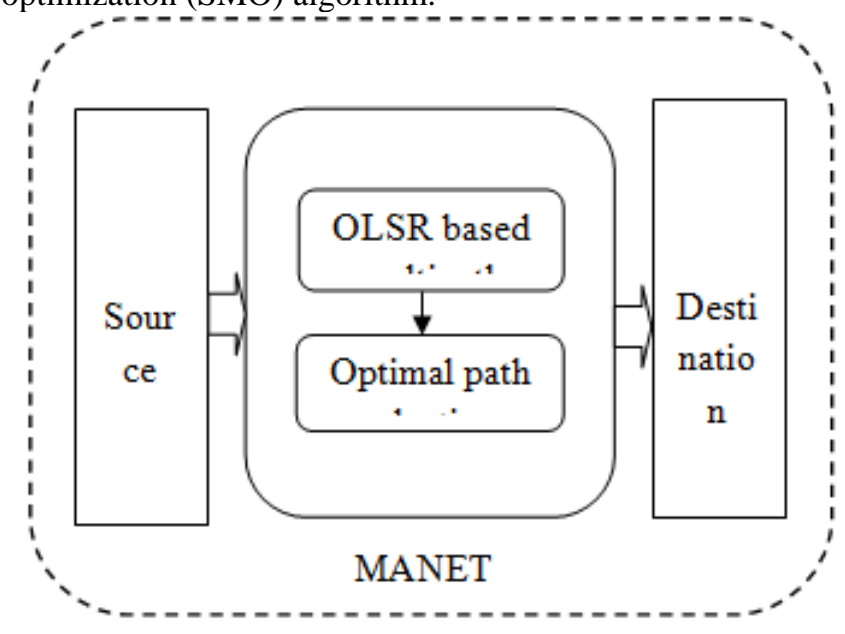

Figure 1: Block diagram of our proposed approach

As shown in Figure 1, MANET has multiple router-like nodes with multiple hosts and wireless communication devices. The OLSR has developed for optimal connections for mobile ad hoc systems. It can also be utilized on additional wireless ad hoc networks. Separate hubs use this topological data to calculate hop locations next to all nodes in the system using short-end hop forwarding routes. The procedure involved in the optimal connection state routing protocol is explained in detail as follows;

\section{A. Optimized Link State Routing Protocol (OLSR):}

OLSR is an optimal connection state table-driven directing convention created for MANET. Optimization depends on a method called Multipoint Relaying. In this, hubs are chosen as MPRs that flood the control messages and cause them to repeat. The Multipoint Relaying procedure decreases the system load and lessens the substance of the control parcels on the grounds that the data sunk into the system is constrained by the data of the MPRs. At the end of the day, a hub that isn't chosen as a MPR won't flood or replay any broadcast messages. Figure 2 shows an example ad hoc system that shows the area of hub A up to two bounce extend.

In regular flooding methods, altogether A-hop neighbors of $\mathrm{H}$, contains $\mathrm{B}$, should return A's regulator packages. But utilizing the Multipoint Relaying method, individual the A, MPR nodes $\mathrm{C}, \mathrm{D}, \mathrm{F}$, also $\mathrm{H}$ hubs (blue nodes) are responsible for transmitting A's control packets. As Figure 1 show, node A's MPR set includes two hop neighbors of node A Every hub in the system periodically sends in addition transmissions regulator manipulations. Since the frequency of these regulator communications is great, it will occasionally cause the loss of some control packets. So there is no need for a re-exchange to lose control packets.
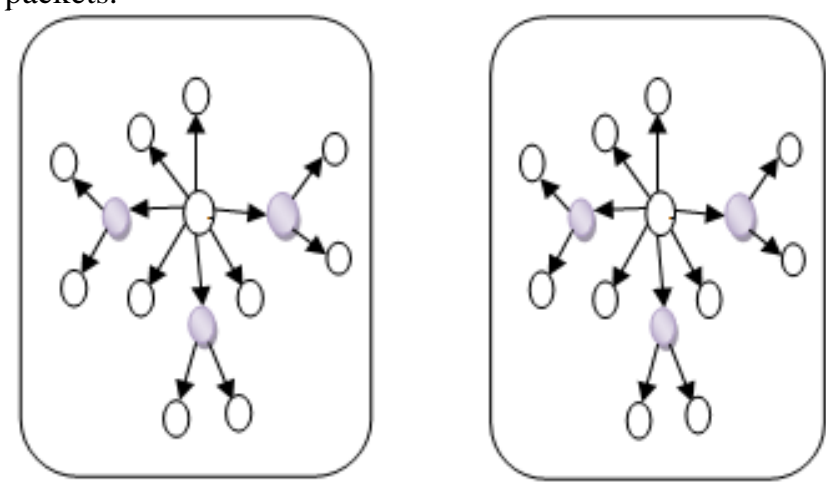

Figure 2: MPR based packet flooding in OLSR

There are two basic categories of regulator communications in the OLSR.

- Hello messages

- Topology Control messages

\section{Hello messages:}

Hop news is occasionally exchanged between a hop neighbors. In this, every hub transmissions its ID then a hop neighbor counters to its neighboring hubs. These messages empower every hub to get data about its environment up to two bounces. At the point when the welcome message is gotten, it records the data of the hello message in its neighbor table. The neighbor table of node $\mathrm{A}$ has the following fields:

- $\quad$ N_addr: Hello message sender ID. This is a hop neighbor of node $\mathrm{A}$.

- N_status: Link Status Code (SYM, ASYM otherwise MPR).

- N2_hoplist: A list of hop neighbors of N_addr (sender of message) which is considered as two hop neighbors of node $\mathrm{A}$.

- N_time: The time this entry is placed in the neighbor table. 


\section{Topology Control messages:}

Some MPR on the system regularly transmissions TC News. TC messages are sent to all hubs in the flooding system's topographic system via MPR nodes. Suppose a node is designated as the MPR hub of particular of its hop neighbors. One-hop sends an MPR node containing a list of neighboring nodes, which are selected as their MPR hub.

A hub connects the data of all received TC communications to determine the topology of the network. MPR hubs are moderate hubs in any way from source to goal. The OLSR protocol can be run freely, however it can likewise work with other protocols to add additional functions, such as linking and security authentication. The process involved in MPR is explained in detail as follows;

\section{B. Multipoint Relays (MPR):}

MPR is one of the main ideas after the OLSR procedure for reducing data overhead. MPR hubs remain the intermediate hubs in the way connecting a source and an objective. Along these lines, MPR hubs assume a significant job in arrange structure:

- MPR hubs spreading TC communications to altogether hubs inside the system.

- Every route from source to goal comprises of MPR hubs. Subsequently, clearly the decision of MPR hubs influences arrange execution. One of the principle explanations behind information misfortune in ad hoc systems is the development of hubs. In spite of the fact that OLSR was created for MANET, the movement of hubs isn't considered in the MPR determination technique. Accept that hubs are separated into two gatherings of versatile and fixed hubs. Our point is to keep portable hubs from picking MPR however much as could reasonably be expected. In doing as such, we expect that the hubs that assume the job of the switch will have increasingly steady and solid courses. Along these lines, no additional control messages are required. Every hub sends its working status in hello messages. Therefore, each node is aware of the mobility of its neighbors. In this, the process involved in the MPR exam is explained in detail as follows;

\section{Multipoint Relays Selection (MPRS):}

In this section the proposed method for selecting a multipoint relay package is described. This method generates the MPR package, which incorporates the base number of jump symmetric neighbors, from which all symmetric do-bounce neighbors can be reached. Host must have data around one and two jump symmetric neighbors to start the computation required for the MPR bundle. Entirely communications are broadcast using hello messages. You can choose to act as MPR with different preferred neighbors than WILL_NEVER in hello communication. Neighbors must be symmetrical to become MPR.

\section{Algorithm for Selecting Multipoint Relay:}

Multipoint Relay Selection algorithm steps are represented as follows;

Step 1: Taking all the balanced one-hop neighbors who want to performance as MPR.

Step 2: Compute the degree of a symmetric neighbor for each neighbor host, dual hubs left since the basis they are computing, and then they do not contain the foundation.
Step 3: Enhance a neighbor symmetric host in the MPR set. If the individual neighbors that can be reached are the two hop neighbors, eliminate the selected host neighbors since the two hop neighbors fixed.

Step 4: On the off chance that there are a couple of more has in the do-hop neighbor set, compute the limit of each jump neighbor, i.e., the quantity of two jump neighbors, I, e the MPR set has not yet been found. Pick the hub with the most favored worth, and if the qualities are the equivalent, the hub with the most elevated number of attainable qualities will be taken. In the event that the feasible limit is the equivalent, take the one with the higher volume determined in the subsequent advance. In the wake of choosing the neighbor for the MPR set, evacuate the two bounce neighbors that are inside reach from the two jump neighbor set.

Step 5:Again the preceding stage pending both hop neighbors are vacant.

Step 6:For optimization, traditional the Host to Increase option in the MPR package. If a host is transported, both hop neighbors are enclosed by the smallest unique host, besides if the host option is reduced than WILL_ALWAYS, the host can be indifferent. To further progress the presentation of the SIP and VoIP signaling system, an OLSR-based cross-layer performance improvement approach is used. The process involved in the cross-layer approach is explained in detail as follows;

\section{Cross-Layer based OLSR Approach:}

A cross-layer method is planned, which helps to maintain the network continuity, resulting in high performance, low latency, low overhead and low battery power consumption that can be used for real-time with real-time communication. In this segment, we scheme a cross-layer method to improve the presentation of SIP and VoIP finished OLSR-depend MANETs. This method uses SIP presentation metrics to preserve the termination processes of SIP calls using SIP logging, call setup, and routing protocol parameters. The OLSR-depend MANET view through the great concentration of mobile hubs utilizing SIP-based VoIP to connect organized, as indicated in Figure 3.

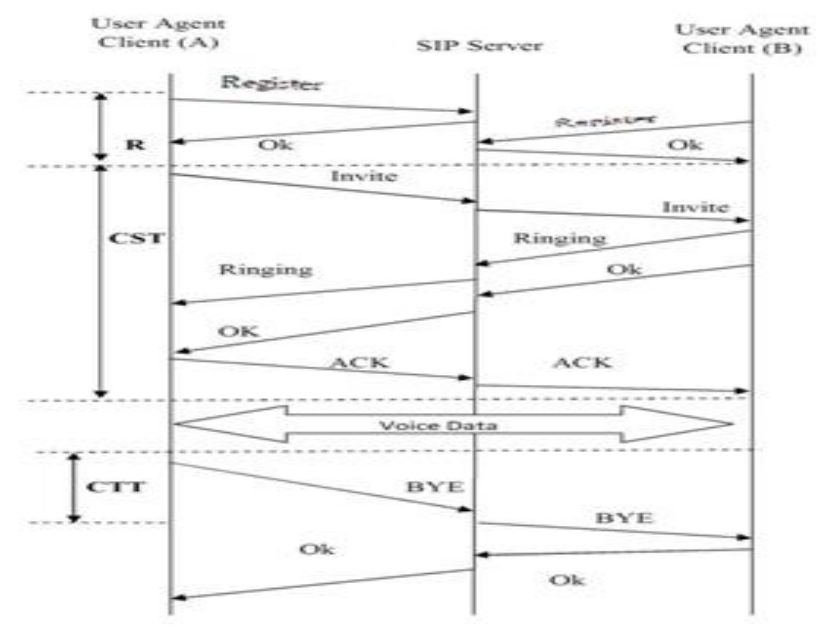

Figure 3: Flow of SIP signaling and RRD, SRD, SDD performance metrics 
Where;

$\mathrm{R} \rightarrow$ register, $\mathrm{CST} \rightarrow$ Call Setup Time, $\mathrm{TT} \rightarrow$ Termination

Time.

This investigation considers a solitary SIP server in this system framework, which gives a SIP record, commencement, and end instrument for SIP calls. Hub A was viewed as the User Agent Client (A) and Node B was the User Agent Client (B). Hubs A and B must be enrolled on the SIP server to recognize their quality just as IP addresses. On the off chance that you need to consider hub A to hub B, the SIP boot messages for the calling framework start flooding between the two hubs by means of the SIP server. At the point when the call is finished, the SIP end message is sent by means of the SIP server. This view utilizes SIP-based VoIP applications as a backup or backup communication network on versatile hubs that help the MANET organize framework. The framework can be utilized for disaster and emergency salvage programs when different correspondences frameworks are lost or broken. This examination thinks about a MANET with moderate spout limit and a wide assortment of movement models.

$\mathrm{RRD}, \mathrm{SRD}$ and SDD performance metrics are represented as follows;

- Registration Request Delay

- Session Request Delay

- Session Disconnect Delay

\section{i) Registration Request Delay (RRD):}

$\mathrm{RRD}$ is utilized to regulate the reaction delay stage of an operator instrument REGISTER application. As noted in Figure 3, RRD enables us to calculate and analyze the user address that generates successful registration requests. RRD production standards must be in milliseconds (ms). This metric is computed utilizing Equation (1), which is mathematically represented as follows;

$R R D=$ Final response time $-\mathrm{Re}$ gister request time

$\mathrm{RRD}$ is measured individual is used for popular records. As the SIP demand load in the system location growths, the RRD assessment similarly escalations. On the other hand, when there are fewer loads inside the system, the RRD importance is also lower.

\section{ii)Session Request Delay (SRD):}

SRD is a measurement intended to identify disabilities or inadequacies that reason delays in reacting to welcome solicitations. SRDs are considered for successful just as bombed session arrangement demands, where the span of achievement and disappointment reactions fluctuates. A basic portrayal of SRD identified with SIP stream is appeared in Figure 3. The SRD is computed utilizing condition (2), which is mathematically represented as follows;

$S R D=$ Time of status indicative response - Time of invite

(2)

\section{iii) Session Disconnect Delay (SDD):}

The SDD is calculated to measure the intermission of time the Session Completion (BYE) communication is directed besides the previous approval (2xx) of the conference conclusion reaction. SDD is utilized to identify deficiencies that can source a delay in completing a session. The SDD effective and failed session is separated and the output esteems are in milliseconds (ms). The SDD is computed utilizing Equation (3), which is mathematically represented as follows;

SDD $=$ Timeof $(2 x x)$ or thimeout - timeof completion message ( BYE)

The performance of the OLSR algorithm is optimized by optimizing multipoint relay nodes using the SMO algorithm. The process involved in the SMO approach is explained in detail as follows;

\section{E. Optimal Selection using Spider Monkey Optimization (SMO):}

The performance of OLSR protocol is enhanced by optimally selecting the multipoint relay nodes using the spider monkey optimization algorithm (SMOA) algorithm. In addition, our proposed algorithm, based on the behavioral framework of the Spider Monkey, aims to improve traditional routing algorithms in efficiency, packet delivery ratio, delay, and packet drop.

SMOA impersonates food seeking performance of spider monkeys. This behavior can be organized in four periods according to the knowledge of FFSS of spider monkeys. Initially, the team evaluates the separation since food, with then starts searching for food. The second stage, the levels of assembly local people with measured since food sources are updated. At a later stage, he renews its position within the local pioneering group. All the group locals are starting to look for food because of the lack of a better level of refreshment by the group's predecessors. In the fourth stage, the global pioneer renews its best position. Due to stagnation the group is divided into smaller subgroups (there is no update on the global precursor position for a predefined time). The LPL has been linked to reduce the incidence of stagnation, which can be distracted by converting the crowd to scavenging. If the global forwarder cannot update the status of the global pioneer limit (GPL), the meeting is divided into smaller subgroups. Negative reviews are being received from LBL and GPL for local and global pioneers to make their choices. This algorithm works in a cross-layer method to the advanced OLSR routing algorithm. The stages of this process are described as follows:

\section{Step 1: Initialization}

To develop an optimal solution at first, the multipoint relay nodes are selected optimally for that purpose SMOA is used. Solution creation i.e., introduction is a significant advance of optimization algorithm that distinguishes the ideal arrangement rapidly. SMOA is demonstrated as pursue. In the hunt space, $\mathrm{N}$ hubs are arbitrarily introduced. Each node represents the solution or optimal multipath routes in the network.

$$
Z_{i}=\left(Z_{i}^{1}, \ldots Z_{i}^{d}, \ldots Z_{i}^{n}\right) \quad \text { for } \quad i=1,2, \ldots N
$$

The obtained solution is given for the next step i.e. fitness evaluation. 


\section{Step 2: Fitness Calculation}

In the wake of creating the solution, the wellness work is assessed and then chooses the best solution. Optimization algorithm for the most part relies upon its fitness function to acquire the best solution. The collection of the fitness is a fundamental feature in SMOA.

$$
\text { Fit }_{p q}(L)=d_{p q}(L)+W_{p q}(L)
$$

Where,

Fit $_{p q}(L) \rightarrow$ Fitness function for transmission of packet $\mathrm{L}$ from node $\mathrm{p}$ to node $\mathrm{q}$,

$d_{p q}(L) \rightarrow$ Time delay required by transmission of packet $\mathrm{L}$ from node $\mathrm{p}$ to node $\mathrm{q}$,

$W_{p q}(L) \rightarrow$ Packet $\mathrm{L}$ transmission from node $\mathrm{p}$ to node $\mathrm{q}$ (the value is integer).

After completing fitness calculation, the obtained results are given for the next step i.e. Update solution.

\section{Step 3: SMOA based Update solution}

This updating phase includes the following stages like local pioneer phase, global pioneer stage, wisdom of stages for global pioneer, leaning phase of local pioneer, decision phase of global pioneer as well as decision phase of local pioneer. These periods of this algorithm are depicted as pursues;

- Local pioneer phase

- Global pioneer phase

- Learning phase of global pioneer

- Learning phase of local pioneer

Decision phase of local pioneer

- Decision phase of global pioneer

These are some of the phases used in spider monkey optimization algorithm. These processes are explained as follows;

Local pioneer phase: At this point, every spider settlement controls its original status depend on the knowledge of local pioneers with local team members. The new status of the spider monkey is computed as follows,

$X_{\text {new }}=\left\{\begin{array}{l}X_{i, d}+r_{1} \times\left(L P_{k, d}-X_{i, d}\right)+r_{2} \times\left(X_{j, d}-X_{i, d}\right) \quad \text { if } r_{1} \geq P R \\ X_{i, d}\end{array}\right.$

otherwise

Where, $r_{1}$ and $r_{2}$ represent the random numeral in the range $[0,1]$ and $[-1,1], L P_{k, d}$ denotes the location of $k^{\text {th }}$ local $\mathrm{d}^{\text {th }}$ dimension

pioneer in the $\quad M_{j, d}$ represents the randomly designated spider monkey from the $j^{\text {th }}$ group in the $d^{\text {th }}$ dimension. PRdenotes the disconcertionamount which manifests the numeral of disconcertion.

Global pioneer phase: The Local Pioneer Phase follows stages called as the global Pioneer Phase. At this point, the spider monkeys renew their status depending on the involvement of local team fellows with global pioneers. The original status of the spider monkey is computed as follows,

$$
X_{n e w_{i, d}}=X_{i, d}+r_{1} \times\left(G P_{d}-X_{i, d}\right)+r_{2} \times\left(X_{j, d}-X_{i, d}\right)
$$

Here, $G P_{d}$ denotes the location of global pioneer in the $d^{\text {th }}$ dimension. Spider monkeys are restructured their location depend on the subsequent possibility cost. This possibility is computed through their qualification. The explanation through the improved possibility updates its location utilizing (7). This possibility $\left(P r_{i}\right)$ importance is measured as purses:

$$
\text { Probability }_{i}=\frac{\text { Fit }_{i}}{\max -\text { Fit }} \times 0.9+0.1
$$

Where, Fit $_{i}$ denotes the fitness assessment of $i^{\text {th }}$ spider monkey, max-Fit denotes the determined fitness assessment in the collection.

Learning phase of global pioneer: In this stage, the situation of global pioneers is refreshed and the spider monkey with the best wellness esteem in the populace is chosen as the global pioneer. In the event that the situation of the global pioneer isn't refreshed, at that point as far as possible check which tallies the quantity of nearby best arrangement is expanded by 1 .

Learning phase of local pioneer: In this stage, the situation of gathering individuals is refreshed and the part with the best wellness esteem in the gathering is chosen as the neighborhood pioneer. On the off chance that the situation of the neighborhood pioneers isn't refreshed, at that point as far as possible check which tallies the quantity of global best solution is expanded by 1 .

Decision phase of local pioneer: If local pioneer amount is superior the local pioneer bound, at that point the situation of all gathering individuals are refreshed haphazardly or dependent on the data of nearby pioneer and global pioneer. The new position of the spider monkey is determined as pursues,

$$
X_{\text {new }_{i, d}}=X_{i, d}+r_{1} \times\left(G P_{d}-X_{i, d}\right)+r_{1} \times\left(X_{i, d}-L P_{j, d}\right)
$$

From this condition (9), it is vacant that the location of the spider monkey is updated towards the location of global pioneer.

Decision phase of global pioneer: At this point, if the global limit number is greater than the global forward limit, the populace is separated into extreme unimportant collections. At this point the local forward limit is initialized to excellent the local collection precedent for each group. If the status of the global pioneer is not updated, then the global pioneer brings all the groups composed with procedures a collection.

\section{Step 4: Termination Phase}

If an optimal solution is executed and the arrangement that has the greatest fitness assessment is selected, the algorithm stops its operation only if it is determined as the best solution. 


\section{RESULT AND DISCUSSION:}

In this section, the performance of the proposed approach is evaluated in terms of throughput, packet delivery ratio, delay and packet drop. This planned methodology is actualized in the foundation of NS2.

\section{A) Performance metrics:}

Execution of our planned methodology is assessed utilizing the accompanying measurements.

Packet delay: The delay of system portrays to what extent the system takes to transmit a piece to the goal. The parameter of unit is seconds (s).

Delivery ratio: It is the fraction of the amount of packets established positively with the total quantity of packages transmitted.

$$
\text { Delivery ratio }=\frac{\text { Amountof packets received }}{\text { Amountof packets transmitted }}
$$

Packet drop: It is the measure of packets dropped through the information broadcast.

Energy consumption: Measure of vitality devoured by every node during the transmission. Additionally it is characterized as the distinction between the present vitality and introductory vitality of a node. The parameter of unit is Joule (J).

\section{Energy consumption = Initial energy - current energy}

(11)

Throughput:It is the quantities of information that can be transmit from the sources to the goal every second. The parameter of unit is $\mathrm{kb} / \mathrm{s}$.

$$
\text { Throughput }=\frac{\text { Amountof transmitted data }(\mathrm{kb})}{\text { Transmitted time }(\mathrm{s})}
$$

Network lifetime: It is characterized as the hour of system detachment because of the disappointment of at least one sensor notes. The parameter of units is seconds (s).

\section{B) Comparative Analysis:}

In this area, our planned strategy SMO-OLSR is contrasted and the current work OLSR. Figure 4-8 show that bundle delay, delivery ratio, energy consumption, throughput just as system lifetime of our planned for differing nodes. This is graphically represented as follows;

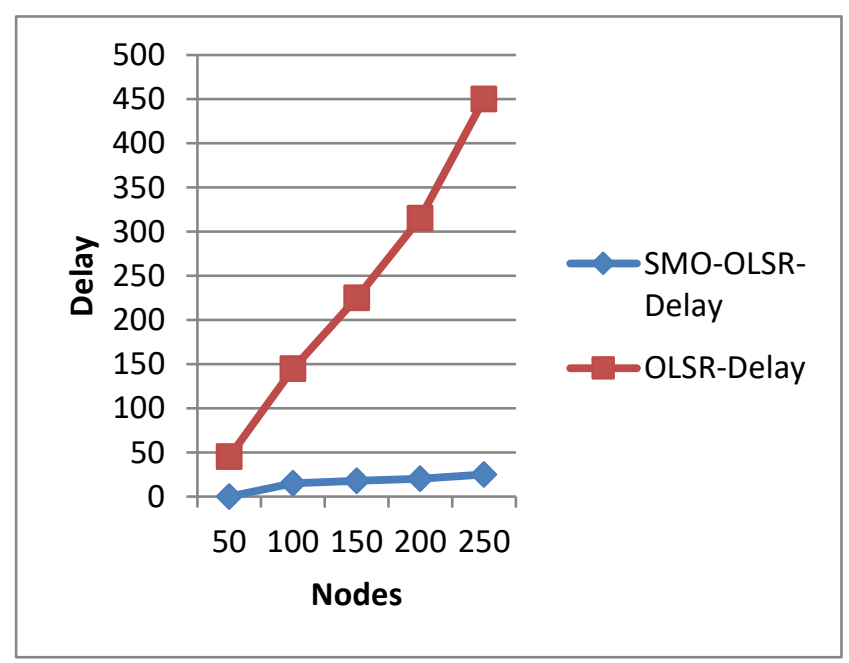

Figure 4: Node Vs Delay

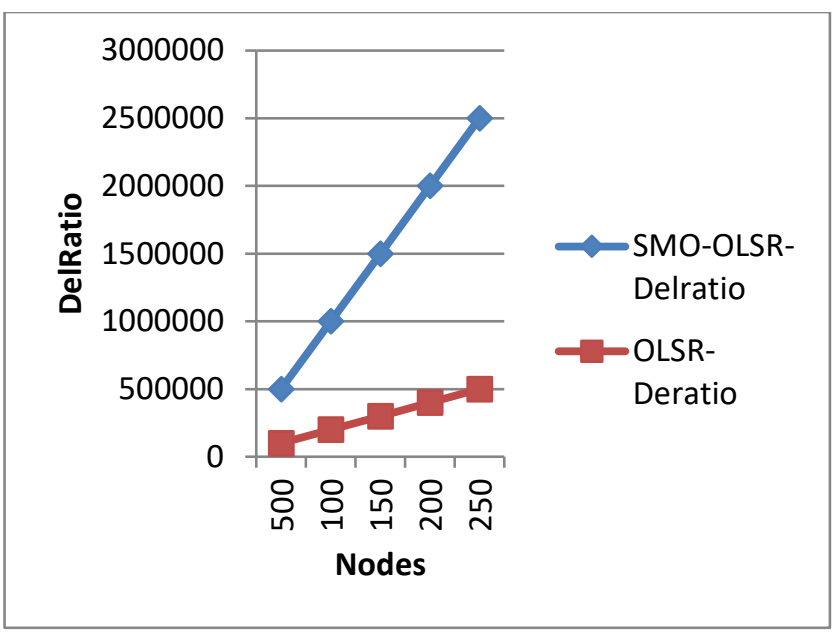

Figure 5: Node Vs Delivery Ratio

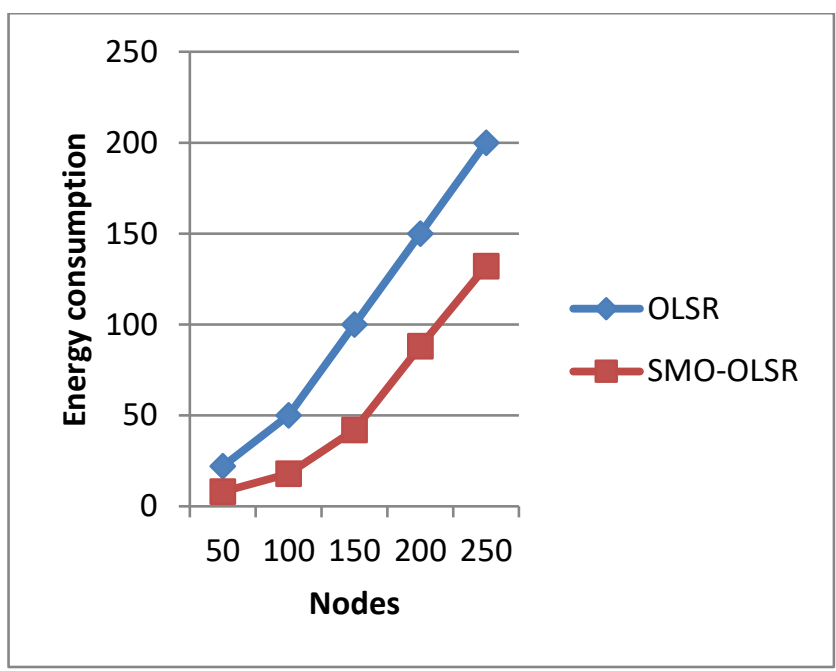

Figure 6: Node Vs Energy

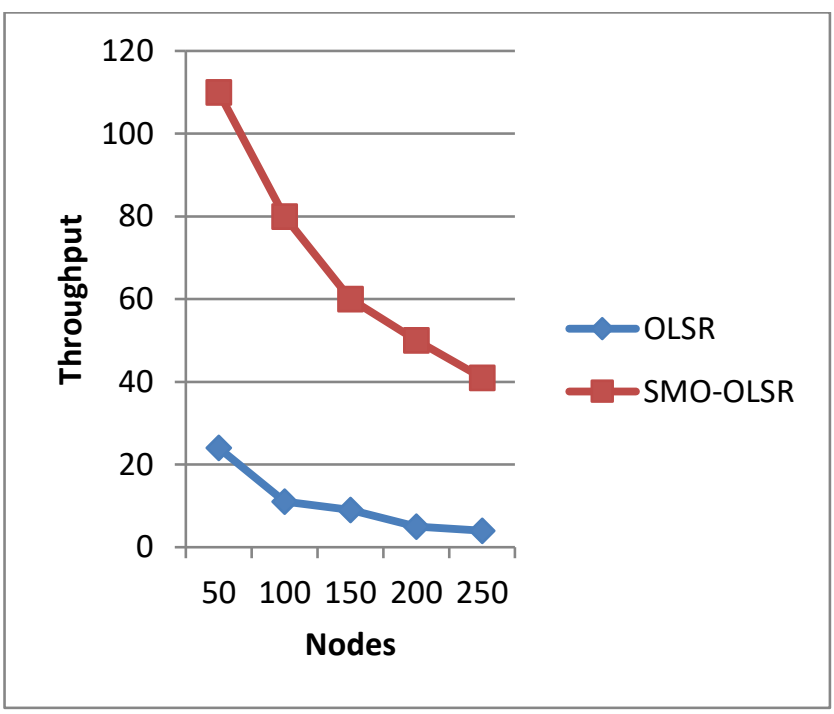

Figure 7: Node Vs Throughput 


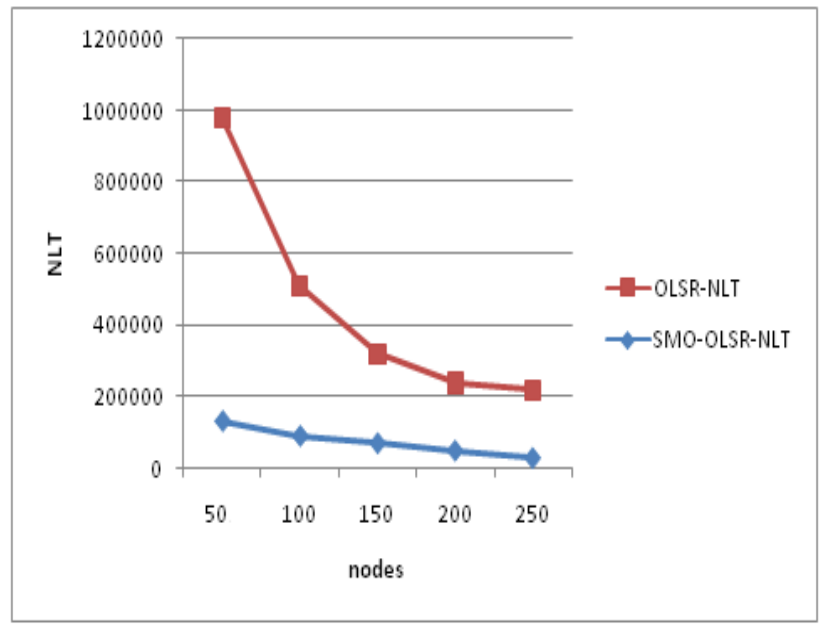

Figure 8: Node Vs NLT

Figure 4 demonstrations the packet delay of our planned system SMO-OLSR, associated to the existing work OLSR. As the quantity of nodes escalations, the network's packet delay also increases. But the packet delay of our planned scheme is low associated to the present work, because it is a cross-layer approach to advanced OLSR-based multipath routing that avoids malicious nodes from the system with reduces the delay for information transmission. Figure 5 demonstrations the delivery ratio of our planned system. Increasing the number of nodes in the network reduces the system delivery rate. But the delivery rate of our planned project SMO-OLSR is developed than the current work. Due to the SMO-OLSR-based routing protocol, information is transmitted to selective multipath routing. Figure 6 demonstrations the energy consumption of the SMO-OLSR of our planned scheme. As the quantity of nodes rises and the energy consumption of the system also raised. But associated to the current work, the optimal path selection using the spider monkey optimization algorithm is significantly reduced by our proposed scheme respectively. The efficiency with system lifetime of our planned methods is shown in Figure $7 \&$ 8, correspondingly. Associated to current work, the efficiency with system lifetime of our planned method is enhanced. By using the spider monkey optimization, an optimal path is selected among the source and the target, thus improving the efficiency with system lifetime of our planned method.

Table. 1 shows the comparison results of previous papers techniques with proposed model in throughout, delay, packet delivery ratio, and energy.

Table 1: Comparison with previous technique and proposed model

\begin{tabular}{|l|c|c|c|c|c|l|}
\hline $\begin{array}{l}\text { References / } \\
\text { Avg }\end{array}$ & {$[16]$} & {$[1]$} & {$[6]$} & {$[2]$} & {$[4]$} & $\begin{array}{l}\text { Proposed } \\
\text { model }\end{array}$ \\
\hline Throughput & - & - & - & 61 & 50 & 92 \\
\hline Delay & - & - & 33 & - & - & 12 \\
\hline $\begin{array}{l}\text { Delivery } \\
\text { Ratio }\end{array}$ & 90 & - & - & - & - & 98 \\
\hline Energy & - & 20 & - & - & - & 80 \\
\hline
\end{tabular}

\section{CONCLUSION}

In this paper, we have given protect and QoS mindful multipath routing in MANET and reproduced our planned technique utilizing the system test system NS2. Energyefficient multipath routes have been chosen utilizing the OLSR algorithm. So as to improve SIP signal execution in MANET, routing parameters must be progressively balanced during SIP forms dependent on the level set for execution improvement measurements to help the SIP signal framework. Accordingly, the effectiveness of the OLSR is to apply between the source and the objective. After the quantity of exchanges, a course may lose its association quality. So the ideal way is chosen utilizing the SMOA. For an efficient communication in hybrid wireless network SMO-OLSR is used. The simulation results demonstrated that our proposed work has better energy efficiency and system lifetime than the current work.

\section{ACKNOWLEDGEMENT}

This Research was supported by Visvesvaraya Technological University, Jnana Sangama, Belagavi 590018 for Grant of financial assistance.

\section{REFERENCE:}

1. Wang, C., Li, J., Yang, Y. and Ye, F., 2018.Combining solar energy harvesting with wireless charging for hybrid wireless sensor networks.IEEE Transactions on Mobile Computing, 17(3), pp.560 576.

2. Salehin, K.M. and Rojas-Cessa, R., 2012.Active scheme to measure throughput of wireless access link in hybrid wired-wireless network.IEEE Wireless Communications Letters, 1(6), pp.645-648.

3. Yang, X., Wang, L., Su, J. and Gong, Y., 2018. Hybrid MAC protocol design for mobile wireless sensors networks. IEEE sensors letters, 2(2), pp.1-4

4. Nita-Rotaru, C., Carbunar, B. and Ioannis, I., 2009. Janus: A framework for scalable and secure routing in hybrid wireless networks. IEEE Transactions on Dependable and Secure Computing, 6(4), pp.295-308.

5. Zhao, S. and Raychaudhuri, D., 2009.Scalability and performance evaluation of hierarchical hybrid wireless networks.IEEE/ACM Transactions on Networking, 17(5), pp.1536-1549.

6. Zoppi, S., Van Bemten, A., Gürsu, H.M., Vilgelm, M., Guck, J. and Kellerer, W., 2018. Achieving Hybrid Wired/Wireless Industrial Networks with WDetServ: Reliability-Based Scheduling for Delay Guarantees. IEEE Transactions on Industrial Informatics, 14(5) pp.2307-2319.

7. Zhang, R., Ruby, R., Pan, J., Cai, L. and Shen, X., 2010. A hybrid reservation/contention-based MAC for video streaming over wireless networks.IEEE Journal on Selected Areas in Communications, 28(3), pp.389-398.

8. Hou, R., Cheng, Y., Li, J., Sheng, M. and Lui, K.S., 2017.Capacity of hybrid wireless networks with long-range social contacts behavior.IEEE/ACM Transactions on Networking, 25(2), pp.834-848.

9. Wang, C., Li, X.Y., Jiang, C., Tang, S. and Liu, Y., 2011.Multicast throughput for hybrid wireless networks under gaussian channel model.IEEE Transactions on Mobile Computing, 10(6), pp.839-852.

10. Tran, T., Nguyen, T., Bose, B. and Gopal, V., 2009.A hybrid network coding technique for single-hop wireless networks.IEEE Journal on Selected Areas in Communications, 27(5), pp.685-698.

11. Ahmed, A. and Shami, A., 2012. RPR-EPON-WiMAX hybrid network: A solution for access and metro networks. Journal of Optical Communications and Networking, 4(3), pp.173-188.

12. Gholami, M.R., Gezici, S. and Strom, E.G., 2012. Improved position estimation using hybrid TW-TOA and TDOA in cooperative networks.IEEE Transactions on Signal Processing, 60(7), pp.37703785. 
13. Wang, Y.C., Peng, W.C. and Tseng, Y.C., 2010. Energy-balanced dispatch of mobile sensors in a hybrid wireless sensor network. IEEE Transactions on Parallel and Distributed Systems, 21(12), pp.18361850.

14. Shin, W.Y., 2011. Refined routing algorithm in hybrid networks with different transmission rates. IEEE Transactions on Communications, 59(5), pp.1242-1246.

15. Han, T.D. and Oh, H., 2011. A looping problem in the tree-based mobility management for mobile IP supported ad hoc networks. Journal of Communications and Networks, 13(4), pp.385-392.

16. Yuan Chai and Xiao-Jun Zeng, "Load- and Interference-Balance Hybrid Routing Protocol for Hybrid Wireless Mesh Network,"University of Manchester, 2019.

17. S. Chaudhari and R. Biradar, "Traffic and mobility aware resource prediction using cognitive agent in mobile ad hoc networks", Journal of Network and Computer Applications, 2016.

18. S. González, W. Castellanos, P. Guzmán, P. Arce and J. Guerri, "Simulation and experimental testbed for adaptive video streaming in ad hoc networks", Ad Hoc Networks, 2016.

19. C. Lal, V. Laxmi, M. Gaur and M. Conti, "Enhancing QoE for video streaming in MANETs via multi-constraint routing", Wireless Netw, 2016.

20. Y. Bao, W. Lei, W. Zhang and Y. Zhan, "QoE collaborative evaluation method based on fuzzy clustering heuristic algorithm", SpringerPlus, vol. 5, no. 1, 2016.

21. S. Chettibi and S. Chikhi, "Dynamic fuzzy logic and reinforcement learning for adaptive energy efficient routing in mobile ad-hoc networks", Applied Soft Computing, vol. 38, pp. 321-328, 2016.

22. DEMIN GAO, SHUO ZHANG, "Row Bee: A Routing Protocol based on Cross-Technology Communication for Energy-Harvesting Wireless Sensor Networks," 2018 IEEE. Translations and content mining.

\section{AUTHORS PROFILE}

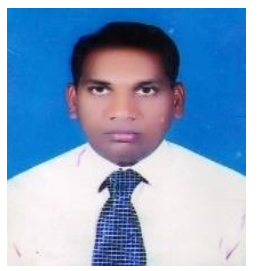

Steven Raj N, received B.E Degree in CSE from REC Bhalki Karnataka. and M.Tech from Poojya Dodappa Appa College of Engineering, Gulbarga Karnataka. Pursuing Ph.D from VTU Belagavi. Presently working as Assistant Professor CSE Department GNDEC Bidar Karnataka.

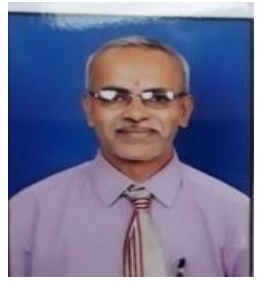

Dr.Dhananjay Maktedar received B.E. from REC Bhalki Karnataka , M.Tech from Sri Jaya Cham Rajendra College of Engineering Mysore Karnataka and completed Ph.D from JNTU Hyderabad in the year 2015,Presently working as a Professor and Head of Computer Science and Engineering Guru Nanak Dev Engineering College, Bidar Karnataka, 22 years of academic Experience and supervising Ph.D Students under VTU University Belagavi, Interested in Research on Image Processin, Pattern Reorganization and WSN. Published papers in various Journals. 